\title{
Erratum: The One-Site Distribution of Gibbs States on Bethe Lattice Are Probability Vectors of Period $\leqslant 2$ for a Nonlinear Transformation ${ }^{1}$
}

Eric Goles and Servet Martínez ${ }^{2}$

Received March 7, 1995

The convex model of automata networks as well as Theorem 2 were first given in ref. 1. Our proof is an alternative one and introduces a Lyapunov operator (see Theorem 1).

\section{REFERENCE}

1. S. Poljak and D. Turzik, On an application of convexity to discrete systems, Discrete Appl. Math. 13:27-32 (1986).

\footnotetext{
${ }^{1}$ This paper appeared in J. Stat. Phys, 52(1/2):267-285 (1988).

2 Departamento de Ingenieria Matematica, Universidad de Chile, Castilla 170/3, Correo 3, Santiago, Chile.
} 